Revue internationale P.M.E.

Économie et gestion de la petite et moyenne entreprise

\title{
Changements de la structure et de la dynamique managériale dans les entreprises devenues publiques Perceptions des employés, des gestionnaires et des PDG
}

\author{
Jocelyn J.-Y. Desroches, Vijay M. Jog et William D. Taylor
}

Volume 4, numéro 3, 1991

URI : https://id.erudit.org/iderudit/1008069ar

DOI : https://doi.org/10.7202/1008069ar

Aller au sommaire du numéro

Éditeur(s)

Presses de l’Université du Québec

ISSN

0776-5436 (imprimé)

1918-9699 (numérique)

Découvrir la revue

Citer cet article

Desroches, J. J., Jog, V. M. \& Taylor, W. D. (1991). Changements de la structure et de la dynamique managériale dans les entreprises devenues publiques :

perceptions des employés, des gestionnaires et des PDG. Revue internationale P.M.E., 4(3), 27-51. https://doi.org/10.7202/1008069ar
Résumé de l'article

Un nouveau sondage, cette fois incluant des gestionnaires et des employés, confirme les effets positifs du financement publique sur leur entreprise et corrobore donc révaluation faite préalablement par leur PDG (Desroches et Jog, 1989). L'étude a été menée au printemps de 1990 auprès d'un échantillon représentatif de l'ensemble des entreprises qui se sont inscrites à la Bourse de Montréal dans le cadre du programme québécois RÉA. Les résultats démontrent une étonnante mais concrète unanimité entre les trois niveaux de perceptions sur le degré et la nature des changements suscités par le nouveau statut de l'entreprise, surtout en ce qui a trait á la formalisation, á la spécialisation, á la décentralisation et á /'utilisation accrue de méthodes analytiques de gestion. 


\title{
Changements de la structure et de la dynamique managériale dans les entreprises devenues publiques \\ Perceptions des employés, des gestionnaires et des PDG
}

\author{
Jocelyn J.-Y. DESROCHES \\ Université du Québec à Montréal* \\ Vijay M. JOG \\ Université Carleton" \\ William D. TAYLOR \\ Université Concordia**
}

\begin{abstract}
RÉSUMÉ
Un nouveau sondage, cette fois incluant des gestionnaires et des employés, confirme les effets positifs du financement publique sur leur entreprise et corrobore donc l'évaluation faite préalablement par leur PDG (Desroches et Jog, 1989). L'étude a été menée au printemps de 1990 auprès d'un échantillon représentatif de l'ensemble des entreprises qui se sont inscrites à la Bourse de Montréal dans le cadre du programme québécois RÉA. Les résultats démontrent une étonnante mais concrète unanimité entre les trois niveaux de
\end{abstract}

* Le professeur Desroches est titulaire d'un doctorat $\mathrm{Ph}$. D. de l'Université de Toronto. Ses domaines de recherche incluent la gestion des PME, les sources alternatives de financement des PME en croissance et la relation entre les gouvernements et le secteur privé. Adresse : Département des sciences administratives, Université du Québec à Montréal, C.P. 6192, succursale A, Montréal (Québec) H3C 4R2.

** Le professeur Jog possède un $\mathrm{Ph}$. $\mathrm{D}$. de l'Université McGill. Ses domaines de recherche et de consultation comprennent la finance corporative, les programmes gouvernementaux, l'entrepreneuriat, les sociétés de capital de risque et l'évaluation d'entreprises. Adresse : Département de finances, Université Carleton, Ottawa (Ontario) K1S 5B6.

*** Le professeur Taylor possède un $\mathrm{Ph}$. D. de l'École des H.E.C. de l'Université de Montréal. Ses domaines de recherche et de consultation se rapportent au management stratégique, et aux systèmes et processus de planification stratégique dans les petites et moyennes entreprises. Adresse : Département de Management, Université Concordia, Montréal (Québec) H3G 1M8.

Les auteurs remercient aussi l'évaluateur anonyme de même que le directeur de la Revue pour leurs commentaires éclairés qui ont conduit à des précisions judicieuses dans le texte, surtout en ce qui concerne les effets de taille de l'entreprise. 
perceptions sur le degré et la nature des changements suscités par le nouveau statut de l'entreprise, surtout en ce qui a trait à la formalisation, à la spécialisation, à la décentralisation et à l'utilisation accrue de méthodes analytiques de gestion.

\section{ABSTRACT}

A new survey, this time including managers and employees, confirms the positive effects of going public for smaller enterprises, thus corraborating an earlier study of Desroches and Jog (1989) which only looked at the perceptions of CEO's. This study was undertaken in the Spring of 1990 using a representative sample of firms which went public on the Montreal Stock Exchange through the Quebec Stock Savings Plan. The results show a clear consensus amongst the three different levels on the degree and nature of the changes a new public status has meant. Structural changes took place with respect to formalization, specialization, decentralization and the use of analytical decision making techniques.

\section{RESUMEN}

Una nueva encuesta, esta vez incluyendo a Gerentes y Empleados, confirma ios efectos positivos del financiamiento público para las empresas, pequeras y apoya por consiguiente, la evaluación hecha anteriormente con los Directores (Desroches y Jog, 1989).

El estudio fue realizado en la primavera de 1990, con una muestra representativa del conjunto de empresas que se inscribieron en la Bolsa de Montreal en el marco del programa «REA » de la Provincia de Quebec, Canadá.

Los resultados demuestran una unanimidad sorprendente entre la percepción de los tres niveles, sobre el grado y la naturaleza de los cambios introducidos por el nuevo estatus publico de la empresa, particularmente en lo que se refiere a la formalización, a la especialización, a la decentralización, y a la mayor utilización de los métodos analíticos de gestión.

\section{Introduction}

Un problème important auquel toutes les PME font face est celui d'obtenir le financement nécessaire au développement de leur entreprise. Dernièrement, de plus en plus de recherches ont été faites sur des sujets tels les facteurs qui amènent les entrepreneurs à choisir le financement public, les effets de ce genre de financement sur les individus qui contrôlent ces entreprises, et les changements sur le plan du rendement et du fonctionnement des PME devenues publiques. 
Le but du présent travail est de rapporter les résultats d'une étude récente qui visait à mesurer l'ampleur des effets d'un premier appel à l'épargne publique (AÉP) sur la gestion de 97 firmes enregistrées à la Bourse de Montréal. Nous examinerons plus particulièrement les changements survenus au regard de la structure organisationnelle et la prise de décision suivant le passage au financement public. Par exemple, nous chercherons à répondre aux interrogations suivantes :

1. La structure de ces entreprises est-elle devenue plus formelle et plus spécialisée?

2. Le processus de prise de décision a-t-il changé avec le financement public de ces entreprises?

3. Les PDG, gestionnaires et employés perçoivent-ils que la prise de décision est devenue plus décentralisée et plus analytique ?

Plus précisément, nous analyserons les perceptions de trois acteurs principaux, (soit le PDG lui-même, les gestionnaires intermédiaires et les employés) sur les transformations dans la gestion de l'entreprise devenue publique sur les plans de la formalisation de la spécialisation de la prise de décision et des techniques d'analyse.

\section{Analyse de la littérature}

Jusqu'à présent, le sujet spécifique des effets du financement public sur les organisations de moindre taille a fait l'objet de très peu de recherche. Cependant, les observations de la théorie organisationnelle et de la littérature entrepreneuriale ont été très utiles pour déterminer les dimensions de la structure organisationnelle et tracer les correspondances entre les différentes structures et stratégies d'une entreprise et son rendement. (Pugh et al, 1967 ; March and Simon, 1958 ; Mintzberg, 1979 ; Marsh and Mannari, 1981 ; Gooding and Wagner, 1985).

La notion selon laquelle les organisations traversent des phases et des cycles de développement est bien établie dans la théorie de gestion. Depuis l'œuvre de Chandler 1962, et les travaux par la suite de plusieurs auteurs (dont Thain, 1969; Churchill et Lewis, 1983; Miller et Friesen, 1983; Covin et Slevin, 1988), plusieurs auteurs ont appuyé le postulat que le développement d'une organisation peut être tracé selon sa complexité stratégique - le rayonnement de ses produits/marchés, sa portée géographique, et le genre d'activités internes nécessaires à la génération de ses produits (Kuhn, 1982; Sandberg, 1986 ; Hofer et Sandberg, 1987). L'âge et la taille d'une entreprise favorisent 
l'expansion de ses activités, et la tendance à ajouter des unités spécialisées croît dans la même mesure (Scott, 1971 ; Toulouse, 1980 ; Miller et Toulouse, 1986 ; Covin et Slevin, 1989).

La première phase structurelle que traverse une organisation est commune à tous les modèles de développement organisationnel. Cette phase est bien exposée par Mintzberg (1979), qui nomme cette configuration « structure simple ». Le point le plus saillant de la structure simple est que, durant cette phase, la plupart des dimensions structurelles de l'organisation restent sans élaboration. En général, les spécialistes sont peu nombreux, la division du travail est très approximative, et les unités d'opération sont mal différenciées. Un autre élément clé de cette étape est le minimum de formalisation en ce qui concerne la planification de l'entreprise et la formation des cadres et des employés.

Mintzberg remarque que la surveillance directe est le dispositif clé de coordination dans les organisations de moindre taille, étant donné que le propriétaire/gestionnaire détient la majeure partie du pouvoir dans l'entreprise. Ceci résulte en un processus de prise de décision très centralisé, avec un pouvoir très étendu pour le propriétaire-dirigeant qui participe et gère lui-même presque toutes les activités de l'entreprise. La communication demeure néanmoins informelle, fréquente et partagée parmi les membres de l'organisation, au cours de cette étape de développement de l'entreprise ; le point de vue qui prédomine demeure toutefois celui du propriétaire.

Qu'est-ce qui fait progresser une organisation au-delà de la structure simple vers la prochaine phase de développement organisationnel ? Évidemment, toute transformation de son environnement fera naître des impulsions vers une nouvelle forme de structure. Si l'environnement change pour inclure de nouveaux partenaires, chacun souvent avec ses propres conceptions et modes de gestion, toutes les décisions ne pourront plus être contrôlées par une seule personne, et une décentralisation de l'entreprise deviendra nécessaire. L'arrivée de nouveaux actionnaires financiers qui exigent, par exemple, un certain niveau de participation à l'orientation et à la gestion de l'entreprise, fait ressortir le besoin (qui serait alors créé) de recevoir plus d'informations et d'établir une meilleure communication. Si par ailleurs, le processus de production d'un nouveau produit ou la fabrication en plus grande quantité d'un produit populaire existant exigeait l'implantation d'une technologie nouvelle et une collaboration plus étroite entre les spécialistes de la fabrication, de la vente et du contrôle budgétaire, une structure administrative plus complexe deviendrait alors indispensable. De même, la situation de dépendance de l'entreprise envers un seul individu ne pourrait plus continuer si les prêteurs ou les investisseurs possibles voyaient que la succession éventuelle de l'entreprise allait poser des problèmes. Une telle situation mènerait à l'affectation d'un commandant en second ou d'un nouveau gestionnaire senior dans l'entreprise à qui seraient confiées certaines responsabilités par les nouveaux actionnaires. 


\section{Études traltant des effets d'un AÉP}

Les effets d'un appel à l'épargne publique (AÉP) sur les organisations de moindre taille est un sujet qui a, en général, suscité très peu d'intérêt du côté de la recherche. Cependant, dans des études antérieures sur des entreprises inscrites en bourse, Desroches et Jog $(1989 ; 1991)$ ont abordé ce sujet, ainsi que d'autres thèmes reliés à la décision d'un entrepreneur de solliciter des fonds publics. Dans leur première publication, Desroches et Jog (1989: tableaux 2 et 3) montrent que les changements les plus importants à l'interne sont survenus sur le plan de la restructuration de l'entreprise. Ces changements, selon les PDG qui ont vécu l'expérience, se sont surtout fait sentir par un accroissement de la fonction managériale (dans $79 \%$ des cas), par l'apparition de nouvelles divisions (dans $54 \%$ des cas) et par l'apparition de nouveaux postes et de nouvelles fonctions (dans $39 \%$ des cas). Et de ces trois changements spécifiques, $59 \%$ des répondants ont jugé l'accroissement de la tâche managériale comme étant le changement le plus marqué.

Bien qu'utile à titre de résultats préliminaires, la recherche de Desroches et Jog était néanmoins limitée sous deux aspects : le premier, par le fait que leur questionnaire s'adressait uniquement aux PDG ; le deuxième, à cause du caractère général des phénomènes étudiés. Ainsi le concept "Structure " fut défini de façon plutôt vague comme étant un des éléments clés du fonctionnement interne de l'entreprise, définition qui, tout en étant juste, donnait peu d'information spécifique concernant le genre de changement concrétisé.

Dans le but de corriger ces faiblesses et d'assurer la validité des résultats obtenus, Desroches, Jog et Taylor (1991) dans une étude commencée en 1988 et récemment complétée, ont d'une part, opérationnalisé et mesuré le concept général de "fonctionnement interne » à plusieurs niveaux spécifiques dont ceux de formalisation, de spécialisation, de prise de décision et de techniques d'analyse. De plus, ils ont interrogé non seulement les PDG, mais aussi les gestionnaires et les employés des mêmes entreprises sur le degré de changement perçu depuis le changement du statut de l'entreprise, (soit de privée à publique).

La méthodologie et les résultats qui suivent, visent donc à préciser brièvement la démarche de recherche suivie, et à analyser les résultats qui supportent et valident les effets positifs du financement public sur l'entreprise, obtenus lors de l'étude de Desroches et Jog, (1989). 


\section{Méthodologie}

Les démarches suivantes ont été effectuées afin de préparer les questionnaires, établir la population, sélectionner l'échantillon de l'étude et choisir les méthodes d'analyse statistique'.

En ce qui concerne l'instrument de collecte d'information, trois questionnaires, basés sur la documentation existante et l'expérience acquise lors de nos recherches antérieures dans le domaine, furent préparés, testés et administrés séparément aux PDG, gestionnaires et employés des entreprises devenues publiques. Les questionnaires comportaient plusieurs sections, et demandaient aux répondants de comparer le degré de formalisation, de spécialisation et de décentralisation de leurs entreprises, ainsi que l'utilisation de techniques analytiques à l'intérieur de celles-ci, avec la situation qui existait avant le passage au financement public. Trois questionnaires différents furent préparés afin d'accommoder les divers groupes visés par l'étude, bien que chaque questionnaire comportait essentiellement les mêmes questions et se référait aux mêmes concepts ${ }^{2}$.

Notre population, se composait de firmes devenues publiques et qui étaient donc inscrites à la Bourse de Montréal par le biais du Programme Régime d'Épargne-Actions (RÉA) du gouvernement du Québec ${ }^{3}$. Une fois ces entreprises répertoriées, il a fallu relever celles où le PDG en poste actuellement était toujours le même qu'au moment où la firme était devenue publique ; celles pour qui ce n'était pas le cas furent retirées de la liste.

Enfin, un troisième critère s'ajoutait, il s'agissait de la limite maximale acceptable des recettes totales annuelles qui ne pouvaient dépasser 100 millions de dollars canadiens. Cette limite, bien que légèrement élevée pour la plupart de nos entreprises de grosseur moyenne et le focus de cette étude sur les

1. Le lecteur intéressé à de plus grandes précisions au sujet de la méthodologie ou pour toute autre information additionnelle, peut en faire la demande aux auteurs ou se référer au Rapport de recherche complet sous Desroches, Jog et Taylor (1991), dans les références bibliographiques.

2. D'autres questions (non soulevées dans cet article) concernaient le statut de l'entreprise, les communications internes et externes, les rapports patron/ouvrier, le degré général de satisfaction, et la satisfaction concernant la décision de devenir publique.

3. Le programme RÉA, établi en 1979 par le gouvernement du Québec, visait d'une part, à faciliter l'accession en bourse d'entreprises québécoises de taille restreinte (c'est-à-dire avec un capital net ou avec une valeur nette égale ou inférieure à 25000000 \$) et d'autre part, à encourager les Québécois à investir dans les titres de ces entreprises. Ce programme a connu ses bonnes années de 1982 jusqu'au krach boursier de 1987. 
changements structurels et stratégiques perçus par les CEO, les gestionnaires et les employés nous ont permis de conserver le maximum de firmes dans une limite acceptable. Néanmoins, $75 \%$ de nos répondants ont déclaré des ventes inférieures à 60 millions de dollars et une valeur des actifs de moins de 50 millions de dollars au cours de la dernière année fiscale disponible, soit 1988 pour la plupart des entreprises ${ }^{4}$.

Après avoir en plus éliminé de la recherche les entreprises qui avaient fait faillite, il restait 97 entreprises qui se conformaient aux critères de l'étude. Le contact avec chacune de ces entreprises a d'abord été établi par téléphone et ensuite par lettre sollicitant sa participation. Après des efforts répétés et systématiques, en personne et par la poste, 55 des 97 entreprises constituant la population totale de l'étude ont accepté et participé à l'étude, soit un taux de réponse de $57 \%$. De ces 55 entreprises, $64 \%$ appartenaient au secteur industriel, et $36 \%$ au secteur des services. Ces entreprises comptaient en moyenne 10 ans d'activité.

En ce qui concerne les répondants comme tels, - notre véritable unité d'analyse -, 41 PDG, 77 gestionnaires et 116 employés ont rempli le questionnaire. Enfin, puisque les entreprises elles-mêmes ont choisi leurs répondants parmi les individus qui faisaient déjà partie de l'organisation avant l'appel à l'épargne publique, il est plausible de croire que les gestionnaires et les employés sélectionnés pour répondre aux questionnaires avaient une attitude plus ou moins positive envers l'entreprise.

En ce qui concerne l'analyse, les données recueillies ont été codées et analysées au moyen de tests standard paramétriques et non paramétriques afin de déterminer la signification statistique de la répartition des réponses entre les PDG, les gestionnaires et les employés (les trois groupes de répondants) sur leur perception des changements survenus concernant plusieurs variables de changements.

\section{Changements structurels suivant un AÉP - Les résultats}

Le postulat de base sous-jacent à la recherche était qu'un premier appel à l'épargne publique entraînerait des changements organisationnels et de la dynamique managériale de l'entreprise. La recherche antérieure de Desroches et Jog (1989) de même que notre revue de la littérature sur le développement organisationnel

4. Voir les sections 5 et 6 ci-après pour plus de précisions. 
postulent qu'une première émission d'actions amène des ressources additionnelles (financières et autres) qui favorisent la croissance de l'entreprise qui, en retour, conduira à des changements aux niveaux dans la structure et le fonctionnement de l'entreprise.

Dans cet article-ci, nous présentons les changements structurels et fonctionnels observés une fois l'entreprise devenue publique ${ }^{5}$, et cela sur les quatre plans suivants :

1. formalisation, ou l'emploi de documents formels observé pour sept catégories.

2. spécialisation, ou la division et la répartition de tâches spécifiques dans l'accomplissement de diverses fonctions.

3. décentralisation, ou le degré d'implication des individus dans le processus de prise de décisions touchant trois secteurs stratégiques et trois secteurs operationnels.

4. prise de décision analytique, ou l'utilisation de techniques systématiques dans l'analyse de sujets reliés au contrôle gestionnaire, au rendement individuel et d'unité, et à la performance stratégique.

\subsection{Formalisation}

Afin de mesurer le degré de formalisation atteint dans l'entreprise, les répondants (les PDG, les gestionnaires et les employés) furent interrogés sur l'utilisation d'une liste de sept catégories de documents officiels avant et après que l'entreprise ne devienne publique.

5. En fait, il est plausible, et même probable, qu'un certain degré de développement au regard de la taille de l'entreprise ait apporté certains changements organisationnels avant que l'entreprise ne devienne publique. L'hypothèse que nous faisons ici toutefois, est que les changements les plus importants touchant la structure et la dynamique managériale ont été conséquents au financement public ou, du moins, perçus comme tels par les répondants des trois niveaux. Ainsi, aucun des 234 répondants n'a soulevé la question avec nous, ce qui pourrait tendre à confirmer notre hypothèse, quels que soient la taille et le rendement de l'entreprise ! (voir les sections 5 et 6 ci-après) 
TABLEAU 1

Pourcentage d'utilisation de documents officiels selon les répondants et le statut de l'entreprise

\begin{tabular}{|c|c|c|c|c|c|c|c|c|}
\hline \multirow[b]{2}{*}{ Documents officiels } & \multirow[b]{2}{*}{ Niveau } & \multicolumn{3}{|c|}{ Avant d'être publique } & \multicolumn{4}{|c|}{ Maintenant } \\
\hline & & Oui & Non & $\begin{array}{l}\text { Ne } \\
\text { sais } \\
\text { pas }\end{array}$ & Oui & Non & $\begin{array}{l}\text { Ne } \\
\text { sais d' } \\
\text { pas }\end{array}$ & $\begin{array}{l}\% \\
\text { l'accrois- } \\
\text { sement } \\
\text { du oui }\end{array}$ \\
\hline $\begin{array}{l}\text { 1. Contrat d'embauche } \\
\text { écrit }\end{array}$ & $\begin{array}{l}\text { PDG } \\
\text { Gestionnaires } \\
\text { Employés }\end{array}$ & $\begin{array}{r}35 \\
29 \\
26\end{array}$ & $\begin{array}{l}60 \\
66 \\
58\end{array}$ & $\begin{array}{r}5 \\
5 \\
16\end{array}$ & $\begin{array}{l}67 \\
48 \\
43\end{array}$ & $\begin{array}{l}33 \\
45 \\
35\end{array}$ & $\begin{array}{r}\overline{7} \\
22\end{array}$ & $\begin{array}{l}48 \\
40 \\
39\end{array}$ \\
\hline $\begin{array}{l}\text { 2. Livret d'informations } \\
\text { ou prospectus sur des } \\
\text { sujets tels la sécurité } \\
\text { au travail, etc. }\end{array}$ & $\begin{array}{l}\text { PDG } \\
\text { Gestionnaires } \\
\text { Employés }\end{array}$ & $\begin{array}{r}34 \\
32 \\
29\end{array}$ & $\begin{array}{l}63 \\
60 \\
56\end{array}$ & $\begin{array}{r}3 \\
8 \\
15\end{array}$ & $\begin{array}{l}63 \\
58 \\
56\end{array}$ & $\begin{array}{l}37 \\
36 \\
31\end{array}$ & $\begin{array}{r}- \\
5 \\
13\end{array}$ & $\begin{array}{l}46 \\
45 \\
48\end{array}$ \\
\hline
\end{tabular}

3. Description écrite des tâches pour :

Employés à la production

Commis de bureau

Superviseur

Administrateur

Chef d'entreprise

4. Organigramme

5. Procédures de travail et politiques écrites

6. Plan d'opération pour les prochains 6 à 12 mois
PDG Gestionnaires 34 Employés 25

PDG

Gestionnaires 31

Employés 23

PDG

Gestionnaires

Employés

PDG

Gestionnaires

Employés

PDG

Gestionnaires

Employés

PDG

Gestionnaires

Employés

PDG

Gestionnaires

Employés

PDG

Gestionnaires

Employés

7. Plan à long terme écrit PDG pour les 3 à 5 prochaines années
Gestionnaires
Employés
35

39

31

52

31
29

66

59

58

64

618

$60 \quad 16$

643

$57 \quad 10$

$\begin{array}{lll}23 & 51 & 26\end{array}$

29

69

63

$14 \quad 43$

2674

2458

124

$49 \quad 46$

55

35

33

6931

$25 \quad 75$

1870

124

$51 \quad 18$

$\begin{array}{lllll}5 & 59 & 38 & 3 & 51\end{array}$

$\begin{array}{llll}54 & 43 & 3 & 37\end{array}$

$\begin{array}{lllll}17 & 43 & 40 & 17 & 42\end{array}$

$\begin{array}{llll}52 & 43 & 5 & 46\end{array}$

$\begin{array}{llll}53 & 46 & 1 & 41\end{array}$

$\begin{array}{llll}37 & 41 & 22 & 38\end{array}$

$\begin{array}{llll}50 & 47 & 3 & 34\end{array}$

$\begin{array}{llll}51 & 45 & 4 & 35\end{array}$

$\begin{array}{llll}36 & 35 & 29 & 36\end{array}$

$\begin{array}{llll}51 & 46 & 3 & 43\end{array}$

$\begin{array}{lllll}10 & 44 & 48 & 8 & 39\end{array}$

$\begin{array}{lllll}43 & 24 & 30 & 46 & 42\end{array}$

- $4456-41$

$\begin{array}{lllll}18 & 31 & 51 & 18 & 23\end{array}$

$\begin{array}{lllll}44 & 22 & 30 & 48 & 45\end{array}$

$\begin{array}{lllll}5 & 78 & 20 & 2 & 37\end{array}$

$\begin{array}{llllll}41 & 4 & 83 & 15 & 3 & 34\end{array}$

$\begin{array}{llllll}54 & 13 & 60 & 26 & 14 & 45\end{array}$

$65-83 \quad 17-58$

$\begin{array}{llllll}57 & 4 & 72 & 26 & 3 & 46\end{array}$

\begin{tabular}{l}
$31-955-27$ \\
\hline 515
\end{tabular}

$\begin{array}{llllll}41 & 7 & 83 & 15 & 3 & 37\end{array}$

$\begin{array}{llllll}35 & 34 & 46 & 24 & 30 & 33\end{array}$

$75-6139-59$

$\begin{array}{llllll}0 & 12 & 39 & 50 & 11 & 54\end{array}$

$\begin{array}{llllll}43 & 45 & 20 & 34 & 46 & 40\end{array}$


Les résultats du tableau 1 montrent une augmentation significative du degré de formalisation dans chacun des sept types de documents. Pour presque toutes les catégories, les répondants des trois niveaux rapportent que l'utilisation de documents formels avait doublé (soit près de $50 \%$ ou plus d'accroissement des oui) dans la période suivant l'appel à l'épargne publique.

Dans les points se rapportant aux relations avec les employés, l'emploi d'un contrat d'embauche écrit a presque doublé suivant l'appel à l'épargne publique, avec $67 \%$ des PDG signalant l'utilisation de ce genre de document, comparé à $35 \%$ qui signalaient leur utilisation avant le financement public de leur entreprise. Le degré d'accroissement d'utilisation de livrets d'informations pour les employés a lui aussi augmenté, plus de $45 \%$ dans chacun des trois niveaux.

Dans les points se rapportant aux procédés d'opération et des rapports entre employés et supérieurs, les trois niveaux ont perçu un accroissement d'utilisation important : d'abord, dans la description écrite des tâches, puis dans les procédures de travail et de politiques écrites de même que le recours à un organigramme formel, où près de $80 \%$ des PDG et des gestionnaires disent s'en prévaloir depuis que la firme est publique.

Dans les points se rapportant d la planification strategique, on note une utilisation beaucoup plus substantielle de plan d'opération à court et à long terme, une fois que l'entreprise est devenue publique. Les taux de réponses élevés reliés à ces deux points montrent de plus qu'il s'agit là du travail du PDG principalement et, dans une moindre mesure, des gestionnaires.

Généralement, les résultats de cette portion de la recherche démontrent que, grâce à un passage au financement public, les entreprises ont formalisé leurs opérations, leurs relations avec les employés, et leurs procédés de planification. En réunissant les perceptions de chaque niveau, et en examinant les sept catégories de documents formels et leurs différences d'accroissement réel au tableau 2, il apparait que de 60 à $80 \%$ des entreprises ont commencé à utiliser des documents formels, tandis qu'avant de devenir publiques, seulement 20 à $30 \%$ avaient utilisé de tels documents.

\subsection{Spéclalisation}

Le deuxième domaine de changement structurel chez les PME devenues publiques visé par l'étude fut le degré de spécialisation des postes dans l'entreprise. Nous avons demandé aux répondants de comparer la situation avant et après le recours au financement public en ce qui concernait les tâches spécifiques assignées pour 14 fonctions différentes. Il fut établi qu'un dépassement de la Phase 1 de développement structurelle de l'organisation signifierait un degré plus élevé de spécialisation. 
TABLEAU 2

Changements dans l'utilisation de documents formels Différences en \% des répondants signalant l'usage avant l'AÉP/maintenant

\begin{tabular}{llccc}
\hline & PDG & Gestionnaires & Employés \\
\hline 1. Contrat d'embauche écrit & 32 & 19 & 17 \\
2. Livrets d'information pour les employés & 29 & 26 & 27 \\
3. Organigrammes & 29 & 28 & 27 \\
4. Description écrite des tâches (propre niveau) & 18 & 22 & 24 \\
5. Procédures de travail écrites & 48 & 33 & 23 \\
6. Plan d'opération pour les prochains 6-12 mois & 26 & 31 & 15 \\
7. Plan à long terme & 36 & 21 & 8 \\
\hline
\end{tabular}

Tous les résultats sont significatifs pour un intervalle de confiance $\leq 0,05$.

TABLEAU 3

Changements dans l'existence de postes spécialisés

Différences en \% des répondants rapportant des responsabilités spécifiques dans un domaine avant l'AÉP/maintenant

\begin{tabular}{lccc}
\hline & PDG & Gestionnaires & Employés \\
\hline 1. Relations publiques, publicité et promotion & $49^{*}$ & $40^{*}$ & $24^{*}$ \\
2. Études de marché & $12^{*}$ & $30^{*}$ & $25^{*}$ \\
3. Ventes & 5 & 6 & 10 \\
4. Services à la clientèle & 2 & 9 & 10 \\
5. Contrôle de la qualité & $23^{*}$ & $23^{*}$ & 9 \\
6. Ressources humaines & $21^{*}$ & $28^{*}$ & $28^{*}$ \\
7. Bien-être des employés & 7 & 16 & 19 \\
8. Distribution & 6 & 9 & 1 \\
9. Organisation temporelle du travail & 8 & 7 & 7 \\
10. Gestion des aspects légaux & $21^{*}$ & $23^{*}$ & $20^{*}$ \\
11. Entretien & 2 & 8 & 12 \\
12. Développement de nouveaux produits $(\mathrm{R}-\mathrm{D})$ & $19^{*}$ & $25^{*}$ & $20^{*}$ \\
13. Tenue de livres et préparation des bilans & 5 & 6 & 9 \\
14. Comptabilité du prix de revient & 9 & $26^{*}$ & $18^{*}$ \\
\hline
\end{tabular}

* Significatif pour un intervalle de confiance $\leq 0,05$.

L'analyse des résultats dans ce domaine a démontré qu'un premier appel à l'épargne publique faisait avancer le processus de spécialisation dans l'entreprise. Comme le montre le tableau 3, une augmentation du degré de spécialisation a été perçue pour chacune des 14 fonctions et ce fut l'avis des 
répondants aux trois niveaux de l'entreprise. Le tableau 4 pour sa part facilite un examen plus précis des changements perçus. Il est clair que dans un certain nombre de catégories le taux de spécialisation était déjà assez élevé, en particulier pour les fonctions suivantes (perceptions des PDG concernant la spécialisation des tâches avant le financement public) : tenue de livres et préparation des bilans : $93 \%$; vente aux clients : $88 \%$; services à la clientèle : $81 \%$; entretien des immeubles et de l'équipement : $69 \%$; comptabilité du prix de revient : $67 \%$; contrôle de la qualité et inspection : $67 \%$. Évidemment, le processus de développement au-delà de la structure simple avait déjà commencé dans ces organisations avant qu'elles ne deviennent publiques.

TABLEAU 4

Pourcentage d'emplois ou de services spécialisés selon les répondants et le statut de l'entreprise

\begin{tabular}{|c|c|c|c|}
\hline $\begin{array}{l}\text { Emplois ou } \\
\text { Services spécialisés }\end{array}$ & Perceptions de & $\begin{array}{c}\text { Avant d'être } \\
\text { une entreprise } \\
\text { publique }\end{array}$ & Maintenant \\
\hline $\begin{array}{l}\text { 1. Relations publiques } \\
\text { publicité et promotion }\end{array}$ & $\begin{array}{l}\text { PDG } \\
\text { Gestionnaires } \\
\text { Employés }\end{array}$ & $\begin{array}{l}31 \\
37 \\
48\end{array}$ & $\begin{array}{l}80 \\
77 \\
72\end{array}$ \\
\hline $\begin{array}{l}\text { 2. Acquisition d'informations } \\
\text { sur les marchés, les consom- } \\
\text { mateurs et les concurrents } \\
\text { (études de marché) }\end{array}$ & $\begin{array}{l}\text { PDG } \\
\text { Gestionnaires } \\
\text { Employés }\end{array}$ & $\begin{array}{l}61 \\
37 \\
35\end{array}$ & $\begin{array}{l}73 \\
67 \\
60\end{array}$ \\
\hline 3. Ventes aux clients & $\begin{array}{l}\text { PDG } \\
\text { Gestionnaires } \\
\text { Employés }\end{array}$ & $\begin{array}{l}88 \\
91 \\
72\end{array}$ & $\begin{array}{l}93 \\
97 \\
82\end{array}$ \\
\hline 4. Services à la clientèle & $\begin{array}{l}\text { PDG } \\
\text { Gestionnaires } \\
\text { Employés }\end{array}$ & $\begin{array}{l}81 \\
83 \\
71\end{array}$ & $\begin{array}{l}83 \\
92 \\
81\end{array}$ \\
\hline $\begin{array}{l}\text { 5. Contrôle de la qualité } \\
\text { et inspection }\end{array}$ & $\begin{array}{l}\text { PDG } \\
\text { Gestionnaires } \\
\text { Employés }\end{array}$ & $\begin{array}{l}67 \\
58 \\
55\end{array}$ & $\begin{array}{l}90 \\
81 \\
64\end{array}$ \\
\hline $\begin{array}{l}\text { 6. Embauche, affectation et } \\
\text { formation du personnel }\end{array}$ & $\begin{array}{l}\text { PDG } \\
\text { Gestionnaires } \\
\text { Employés }\end{array}$ & $\begin{array}{l}52 \\
45 \\
38\end{array}$ & $\begin{array}{l}73 \\
73 \\
66\end{array}$ \\
\hline $\begin{array}{l}\text { 7. Bien-être, sécurité et } \\
\text { avantages des employés }\end{array}$ & $\begin{array}{l}\text { PDG } \\
\text { Gestionnaires } \\
\text { Employés }\end{array}$ & $\begin{array}{l}64 \\
59 \\
54\end{array}$ & $\begin{array}{l}71 \\
76 \\
73\end{array}$ \\
\hline $\begin{array}{l}\text { 8. Distribution et livraison } \\
\text { des produits finis }\end{array}$ & $\begin{array}{l}\text { PDG } \\
\text { Gestionnaires } \\
\text { Employés }\end{array}$ & $\begin{array}{l}54 \\
63 \\
68\end{array}$ & $\begin{array}{l}60 \\
72 \\
69\end{array}$ \\
\hline $\begin{array}{l}\text { 9. Planification et horaire } \\
\text { de travail }\end{array}$ & $\begin{array}{l}\text { PDG } \\
\text { Gestionnaires } \\
\text { Employés }\end{array}$ & $\begin{array}{l}64 \\
66 \\
61\end{array}$ & $\begin{array}{l}76 \\
73 \\
68\end{array}$ \\
\hline
\end{tabular}


TABleau 4 (suite)

Pourcentage d'emplois ou de services spécialisés selon les répondants et le statut de l'entreprise

\begin{tabular}{llcc}
\hline $\begin{array}{l}\text { Emplois ou } \\
\text { Services spécialisés }\end{array}$ & Perceptions de & $\begin{array}{c}\text { Avant d'être } \\
\text { une entreprise } \\
\text { publique }\end{array}$ & Maintenant \\
\hline 10. Gestion des aspects légaux & PDG & 60 & 81 \\
et des questions d'assurance & Gestionnaires & 56 & 79 \\
& Employés & 44 & 64 \\
11. Entretien des immeubles & PDG & 69 & 71 \\
et de l'équipement & Gestionnaires & 72 & 80 \\
& Employés & 57 & 69 \\
12. Développement de & PDG & 54 & 73 \\
nouveaux produits et & Gestionnaires & 53 & 78 \\
procédés (R - D) & Employés & 47 & 62 \\
13. Tenue de livre et & PDG & 93 & 98 \\
préparation des bilans & Gestionnaires & 94 & 100 \\
& Employés & 77 & 86 \\
14. Développement, analyse & PDG & 67 & 76 \\
des coûts et revenus & Gestionnaires & 65 & 91 \\
(gestion du prix de revient) & Employés & 59 & 77 \\
\hline
\end{tabular}

La fonction relations publiques, publicité et promotion est celle qui a eu l'augmentation la plus signifiante d'assignation de tâches spécialisées. Par exemple, seulement 31 \% des PDG citaient une assignation spécifique de tâches dans l'accomplissement de cette fonction avant de passer au financement public, tandis que $80 \%$ répondaient que maintenant ces tâches spécifiques étaient assignées. D'autres fonctions ont connu une augmentation considérable du taux de spécialisation : la gestion du personnel (changements de $21 \%, 28 \%, 28 \%$ perçus par PDG, gestionnaires et employés respectivement), le contrôle de la qualité et l'inspection $(23 \%, 23 \%, 9 \%)$, la gestion des aspects légaux et des questions d'assurance $(21 \%, 23 \%, 20 \%)$, l'étude de marchés $(12 \%, 30 \%$, $25 \%)$, le développement de nouveaux produits et procédés (R-D) $(19 \%, 25 \%$, $15 \%)$, et la gestion du prix de revient $(9 \%, 26 \%, 18 \%)$.

Il semblerait que le passage au financement public a permis aux entreprises de concentrer et d'améliorer des aspects de leurs organisation qu'elles ne développaient pas auparavant, et de consacrer leurs effectifs à de nouveaux produits et à des projets d'expansion. L'augmentation de spécialisation dans la gestion des aspects légaux et les questions d'assurance est sans doute reliée aux exigences régulatoires qu'impose le statut d'entreprise publique. 
Il y avait néanmoins certaines fonctions pour lesquelles le degré de spécialisation était perçu différemment par les répondants venant des trois différents niveaux de l'entreprise. En général, les réponses s'accordaient pour montrer la tendance vers une spécialisation accrue ; c'est concernant le point auquel il y avait augmentation de spécialisation que les différences de perception entre les niveaux ressortaient. Pour trois fonctions, les perceptions de changement ont beaucoup varié entre les trois groupes de répondants. D'abord, gestionnaires et employés ont noté une augmentation beaucoup plus importante dans l'assignation de tâches spécialisées pour les fonctions étude de marchés et comptabilité du prix de revient. En ce qui concernait les études de marché, les PDG trouvaient que l'assignation de tâches spécialisées s'effectuait déjà à un plus haut degré avant le financement public, et, conséquemment, n'ont pas perçu un changement aussi significatif. Pour ce qui était des tâches reliées à la comptabilité du prix de revient, le degré de changement a aussi été perçu comme étant plus important par les gestionnaires et les employés. L'accomplissement de ces deux fonctions comprend des tâches et des responsabilités analytiques, ce qui suggère que les PDG, plus que les gestionnaires, percevaient que leurs organisations avaient développé une expertise technique, ou analytique, avant l'appel à l'épargne publique.

Également, un taux beaucoup plus élevé de spécialisation fut remarqué par les employés dans l'assignation de tâches pour la fonction bien-être, sécurité et avantages des employés. Il doit être signalé que leur position dans l'entreprise les rendait plus sensibles à ce genre de changement.

Pour résumer, il est très évident que le processus de spécialisation s'est poursuivi dans ces entreprises dans la période suivant leur appel à l'épargne publique. Le fait qu'il existe beaucoup d'accord dans la perception des répondants venant des trois niveaux de l'organisation donne davantage d'appui aux résultats de l'étude. Ces résultats démontrent également que, pour la majorité des entreprises étudiées lors de cette recherche, la spécialisation est maintenant arrivée à un degré qui assure qu'au moins une personne effectue des tâches spécialisées, à temps plein ou à temps partiel, dans l'accomplissement de chaque fonction.

\subsection{Décentralisation de la prise de décision}

Cette recherche s'est également intéressée aux perceptions des répondants concernant leur degré de participation dans la prise de décisions touchant six secteurs différents, et comment cette participation aurait changé depuis le financement public de leur entreprise. Le tableau 5 résume le degré de décentralisation perçu par les répondants et présente les secteurs investigués. Trois de ces secteurs touchent des questions reliées à l'opération de l'entreprise (gestion du personnel, planification de la production, et publicité et promotion, 
les points 1,2 et 3 ), et les trois autres touchent des questions plutôt stratégiques dans leur aspect temporel (achat d'équipement de production et d'actifs tangibles (point 4), développement de nouveaux produits (point 5), et diversification vers de nouveaux produits et marchés (point 6)).

Pour plus de précision, nous analyserons ci-après les réactions de chacun des répondants sur leur degré de participation perçu dans l'entreprise.

\section{TABLEAU 5}

\section{Changements dans le degré perçu de décentralisation} Niveau auquel les décisions sont prises

Je prends maintenant... (\% réponse)

\begin{tabular}{llrrrrr}
\hline $\begin{array}{l}\text { Décisions } \\
\text { touchant }\end{array}$ & & $\begin{array}{c}\text { Beaucoup } \\
\text { plus de } \\
\text { décisions } \\
\text { qu'avant }\end{array}$ & $\begin{array}{c}\text { Plus de } \\
\text { décisions } \\
\text { qu'avant }\end{array}$ & $\begin{array}{c}\text { Aucun } \\
\text { change- } \\
\text { ment }\end{array}$ & $\begin{array}{c}\text { Moins de } \\
\text { décisions } \\
\text { qu'avant }\end{array}$ & $\begin{array}{c}\text { Beaucoup } \\
\text { moins de } \\
\text { décisions } \\
\text { qu'avant }\end{array}$ \\
\hline 1. L'embauche, & PDG* & 0 & 7 & 66 & 20 & 7 \\
l'affectation et le & Gestionnaires* & 9 & 34 & 46 & 7 & 3 \\
renvoi des travailleurs & Employés* & 10 & 18 & 56 & 7 & 4 \\
2. La priorité des & PDG* & 0 & 7 & 57 & 26 & 10 \\
commandes, échéances & Gestionnaires* & 18 & 22 & 49 & 4 & 4 \\
de livraisons, & Employés & 7 & 22 & 43 & 12 & 4 \\
plans de production & & & & & & \\
3. La promotion & PDG & 10 & 29 & 31 & 26 & 2 \\
et la publicité des & Gestionnaires* & 12 & 24 & 57 & 3 & 3 \\
produits de l'entreprise & Employés* & 9 & 30 & 38 & 10 & 1 \\
4. L'achat d'équipe- & PDG & 2 & 29 & 48 & 14 & 5 \\
ment de production & Gestionnaires* & 19 & 30 & 43 & 5 & 0 \\
et des actifs tangibles & Employés* & 12 & 29 & 35 & 6 & 4 \\
5. Le développement & PDG* & 14 & 33 & 38 & 10 & 3 \\
de nouveaux & Gestionnaires* & 15 & 28 & 51 & 1 & 3 \\
produits & Employés & 9 & 24 & 37 & 13 & 3 \\
6. La diversification & PDG* & 17 & 45 & 38 & -5 & - \\
vers de nouveaux & Gestionnaires* & 12 & 39 & 44 & 3 & 0 \\
marchés géographiques & Employés* & 12 & 32 & 35 & 4 & 2 \\
\hline
\end{tabular}

* Significatif pour un intervalle de confiance $\leq 0,05$ 


\subsubsection{Perception des PDG}

En ce qui concerne les questions d'ordre opérationnel, les résultats de cette étude ont été conformes aux attentes dans deux des secteurs. Les PDG ont rapporté qu'ils avaient décentralisé, ou transféré, la prise de décision vers leurs gestionnaires, spécialement dans les secteurs gestion du personnel et planification de la production. Il suffit de considérer les perceptions des gestionnaires pour vérifier ce changement. Comme nous le verrons plus tard, les groupes administratifs (qui répondent aux PDG dans ces entreprises) conviennent qu'un plus grand nombre de décisions de genre opérationnel ont en effet été accordés à leur niveau. Par exemple, tandis que $27 \%$ des PDG rapportent qu'ils prennent moins de décisions concernant le personnel, $43 \%$ des gestionnaires déclarent qu'ils participent maintenant plus, ou beaucoup plus, à la prise de décisions dans la gestion du personnel.

Significativement, selon les PDG les changements les plus marqués touchaient les décisions dites stratégiques. Plus de $60 \%$ des repondants ont indiqué qu'ils prenaient maintenant plus de décisions concernant la diversification de leur entreprise vers de nouveaux marchés géographiques. Les PDG sont aussi plus impliqués dans le développement de nouveaux produits, avec $47 \%$ indiquant qu'ils prennent maintenant plus, ou beaucoup plus, de décisions concernant de nouveaux produits. Deux raisons peuvent expliquer ces changements dans le degré de décentralisation suivant un appel à l'épargne publique. La première est que les fonds générés ont résulté en un accroissement des activités d'expansion de ces PME. Plus simplement, il y a plus de décisions à prendre sur ces questions. La deuxième est que les PDG, ayant moins de temps à consacrer aux questions d'expansion, ont été obligés de laisser à d'autres le soin de prendre les décisions touchant les questions de type operationnel.

\subsubsection{Perception des gestionnaires}

Les gestionnaires qui ont répondu à notre enquête ont fortement confirmé le mouvement vers une prise de décision plus décentralisée dans leurs organisations. Ils rapportent que non seulement ils sont plus impliqués dans les décisions de type operationnel que les PDG leur ont confiées, mais qu'ils prennent plus de décisions dans tous les autres secteurs aussi. En effet, les changements les plus importants dans la prise de décision, selon les gestionnaires, touchent les questions dites stratégiques, qui visent le long terme, avec $51 \%$ des gestionnaires rapportant qu'ils prennent plus de décisions concernant la diversification, et $43 \%$ déclarant qu'ils prennent maintenant plus de décisions concernant le développement de nouveaux produits. 
Ces résultats appuient solidement le mouvement vers une prise de décision décentralisée. Il faut remarquer que, si les deux niveaux, PDG et gestionnaires, rapportent une augmentation de leur participation dans la prise de décision, ce n'est pas nécessairement contradictoire. Le questionnaire ne précisait pas à quel point un individu devait être seul responsable de la décision, et permettait donc plusieurs interprétations différentes de l'énoncé «prendre une décision ». Par exemple, si le PDG prenait une décision d'endosser une expansion avec l'actif immobilisé, les gestionnaires individuels pouvaient régler les questions plus spécifiques de la décision. Ainsi, $49 \%$ des gestionnaires ont perçu qu'ils prenaient maintenant plus, ou beaucoup plus de décisions concernant des achats d'actif immobilisé, tandis que les PDG indiquaient qu'aucun changement ne s'était produit dans ce domaine.

On a aussi demandé aux gestionnaires si les employés travaillant pour eux prenaient maintenant plus de décisions, afin de découvrir si eux aussi avaient également décentralisé certaines de leurs décisions. Les résultats montrent que $60 \%$ des gestionnaires percevaient que les gens travaillant pour eux participaient plus aux décisions, indiquant une plus importante délégation vers les niveaux moins elevés de l'organisation.

\subsubsection{Perception des employés}

Les réponses des employés concernant le degré d'augmentation du nombre de décisions qu'ils avaient maintenant à prendre sont assez surprenantes. Dans 5 des 6 secteurs, les employés percevaient qu'ils prenaient plus, ou beaucoup plus, de décisions. Étant donné que seulement $60 \%$ des PDG percevaient que leurs employés prenaient plus de décisions, l'envergure des résultats obtenus chez les employés était inattendue. Le seul secteur où les employés ne rapportaient aucun changement était la planification et l'organisation temporelle du travail.

Une raison possible à la perception des employés d'un si haut niveau de décentralisation verticale pourrait être attribuée au questionnaire lui-même. Malheureusement, le questionnaire n'exerçait aucun contrôle dans le domaine de la sélection des employés répondants ; cette tâche fut laissée aux PDG. Une autre condition stipulait que les employés sélectionnés devaient avoir fait partie de l'organisation avant le financement public. Ainsi, il était possible que les employés soient au niveau superviseur ou même gestionnaire de bas échelon. Quoique les résultats obtenus chez les employés semblent appuyer la conclusion générale d'une décentralisation accrue dans les PME étudiées, nous ne pouvons pas tirer de conclusions fermes à partir des réponses fournies par les employés sans avoir plus de données concrètes sur ces individus et leurs responsabilités. 


\section{TABLEAU 6 \\ L'emploi de techniques analytiques \\ \% des répondants qui sont d'accord avec l'énoncé \\ depuis le passage au financement public de leur entreprise...}

\begin{tabular}{lccc}
\hline & PDG & Gestionnaires & Employés \\
\hline $\begin{array}{l}\text { 1. Meilleures techniques pour contrôler } \\
\text { la qualité des opérations }\end{array}$ & 69 & 58 & 53 \\
$\begin{array}{l}\text { 3. Des centres de coût et de profit mieux définis } \\
\text { 5. L'assignation plus précise } \\
\begin{array}{l}\text { de la responsabilité des coûts et profits } \\
\text { Processus plus formel d'évaluation } \\
\text { des performances personnelles }\end{array}\end{array}$ & 81 & 72 & 56 \\
$\begin{array}{l}\text { 7. Systèmes plus exhaustifs d'information } \\
\text { et de contrôle de gestion }\end{array}$ & 64 & 47 & 55 \\
\hline
\end{tabular}

Tous significatif pour un intervalle de confiance $\leq 0,05$

\subsection{Techniques analytiques}

Un autre domaine de changement interne examiné lors de cette recherche fut l'emploi de techniques analytiques par les entreprises étudiées. On a demandé aux répondants s'ils étaient d'accord que leur entreprise disposait de systèmes, de techniques et d'approches plus sophistiqués et plus étendus depuis son passage au financement public.

Comme le montre le tableau 6, plusieurs ont convenu que depuis l'acquisition du nouveau statut public, les entreprises avaient fait un progrès substantiel dans toutes les catégories examinées. Dans l'ensemble, les PDG et les gestionnaires étaient surtout d'accord avec le fait que la définition de centres de profit, et les systèmes de comptabilité des responsabilités étaient devenus plus analytiques. Comme le tableau le démontre, $81 \%$ des PDG et $66 \%$ des gestionnaires étaient d'accord ou fortement d'accord que leur entreprise avait maintenant une assignation plus précise de la responsabilité des coûts et profits à des administrateurs clés (point 1); et $78 \%$ des PDG et $72 \%$ des gestionnaires convenaient que leur entreprise définissait maintenant mieux les centres de coûts et de profits (point 2). À notre avis, cela contribue au sentiment profond chez la direction de l'entreprise d'être beaucoup mieux équipée pour mesurer le rendement des cadres et pour assigner les responsabilités appropriées.

Les autres catégories où il y avait beaucoup d'accord parmi le personnel de direction traitaient également des questions de comptabilité du prix de revient et de systèmes de contrôle de la gestion (point 3 ). Par exemple, $73 \%$ des PDG et $61 \%$ des gestionnaires convenaient qu'ils disposaient maintenant de 
meilleures données sur les coûts, tandis que $69 \%$ des PDG et $58 \%$ des gestionnaires percevaient comme complément à la gestion des coûts, la mise sur pied de meilleures techniques de contrôle et de la qualité des opérations (point 4).

Le mouvement vers une spécialisation accrue des systèmes de comptabilité de la gestion, abordé plus haut, est reflété par un emploi plus répandu de l'analyse dans la prise de décision. Ces résultats confirment également le mouvement vers des systèmes plus formalisés touchant chaque individu personnellement. En effet, les personnes interrogées aux trois niveaux ont noté une tendance vers ces processus plus formels d'évaluation des performances personnelles (avec des degrés d'accord de $64 \%, 47 \%$ et $46 \%$ chez PDG, gestionnaires et employés respectivement (point 5)).

En conclusion, les résultats présentés ci-haut confirment donc que les PDG, les gestionnaires et les employés d'entreprises devenues publiques, ont remarqué une utilisation plus importante de techniques analytiques qu'avant que leur entreprise ne devienne publique. Bien que tous les résultats étaient statistiquement significatifs pour un intervalle de confiance $\leq 0,05$, l'intensité d'accord était un peu moindre pour les gestionnaires, et encore un peu moindre pour les employés. Il convient peut-être de noter que les employés montraient leur plus haut degré d'assentiment au fait que leur entreprise comptait maintenant plus de techniques analytiques pour les assister dans la prise de décision à leur niveau, et qu'il y avait plus de spécialistes qu'ils pouvaient consulter. Ces résultats montrent la préoccupation qu'a ce niveau de bien accomplir son travail dans la hiérarchie, et son besoin d'appui dans un système décentralisé.

\section{La correspondance entre rendement, taille, et changements dans l'organisation}

Étant donné les degrés très importants de changement des structures et des processus de prise de décision dans les entreprises étudiées, il est utile de tenir compte ici des informations additionnelles concernant leur taille et leur rendement financier. Il est possible que quelques-uns de ces changements favorables soient liés à l'agrandissement des entreprises grâce à l'afflux de capital additionnel. Plus spécifiquement, il peut être maintenu que, si toutes les entreprises de cette étude étaient larges et homogènes au départ, les considérations de taille l'emporteraient sur les considérations d'effets du financement public. Il est également possible d'arguer que toutes les firmes étudiées étaient déjà des entreprises à haut rendement, ce qui donne des résultats reflétant seulement les changements qui ont lieu dans les entreprises qui réussissent. 
TABLEAU 7

Distribution des firmes de l'échantillon selon leur actif, 1989

\begin{tabular}{lcrrr}
\hline & \multicolumn{2}{c}{ Groupe $\mathbf{A}^{*}$} & \multicolumn{2}{c}{ Groupe B** } \\
Actif & $\mathbf{n}^{*}$ & $\%$ & $\mathbf{n}^{\circ}$ & $\%$ \\
\hline (M \$) & 3 & 8,8 & 7 & 13,0 \\
PE10 et MQ20*** & 9 & 26,5 & 13 & 24,1 \\
PE20 et MQ30 & 7 & 20,6 & 11 & 20,4 \\
PE30 et MQ40 & 6 & 17,6 & 10 & 18,5 \\
PE40 et MQ50 & 1 & 2,9 & 2 & 3,7 \\
PE50 et MQ60 & 2 & 5,9 & 2 & 3,7 \\
PE60 et MQ70 & - & - & 2 & 3,7 \\
PE70 et MQ80 & 2 & 5,9 & 2 & 3,7 \\
PE80 et MQ90 & 1 & 2,9 & 1 & 1,9 \\
PE90 & 3 & 8,8 & 4 & 7,4 \\
& 34 & 99,9 & 54 & 100 \\
\hline
\end{tabular}

- Firmes dont les répondants provenaient des trois niveaux, PDG, gestionnaires et employés.

- Firmes dont les répondants provenaient d'au moins un des trois niveaux.

*** $\mathrm{PE}=$ Plus grand ou égal à ; MQ = Moins que.

\section{TABLEAU 8}

Distribution des firmes de l'échantillon selon leurs ventes, 1989

\begin{tabular}{lrrrr}
\hline & \multicolumn{2}{c}{ Groupe $\mathbf{A}^{*}$} & \multicolumn{2}{c}{ Groupe B** $^{* *}$} \\
\hline Ventes totales & $\mathbf{n}^{\circ}$ & $\%$ & $\mathbf{n}^{\circ}$ & $\%$ \\
\hline M \$) & 2 & 5,9 & 8 & 14,8 \\
PE10 et MQ20*** & 7 & 20,6 & 10 & 18,5 \\
PE20 et MQ30 & 2 & 5,9 & 3 & 5,6 \\
PE30 et MQ40 & 8 & 23,5 & 11 & 20,4 \\
PE40 et MQ50 & 7 & 20,6 & 8 & 14,8 \\
PE50 et MQ60 & 4 & 11,8 & 5 & 9,3 \\
PE60 et MQ70 & 1 & 2,9 & 3 & 5,6 \\
PE70 et MQ80 & 1 & 2,9 & 2 & 3,7 \\
PE80 et MQ90 & - & - & 2 & 3,7 \\
PE90 & 2 & 5,9 & 2 & 3,7 \\
& 34 & 100 & 54 & 100 \\
\hline
\end{tabular}

- Firmes dont les répondants provenaient des trois niveaux, PDG, gestionnaires et employés.

** Firmes dont les répondants provenaient d'au moins un des trois niveaux.

*** $\mathrm{PE}=$ Plus grand ou égal à ; MQ = Moins que. 
Les informations pertinentes sont basées sur les caractéristiques financières des entreprises étudiées. Ces informations ont été recueillies auprès des 34 compagnies ayant des répondants des trois niveaux (groupe A), ainsi que chez les compagnies dont au moins un des trois groupes de répondants avait rempli le questionnaire (groupe B). Les caractéristiques financières suivantes furent utilisées : total de l'actif, ventes, revenu de l'actif, revenu des actions, et marge d'exploitation. Les deux premières caractéristiques fournissent une indication de la taille des entreprises examinées dans la recherche. Les trois autres caractéristiques fournissent une indication du rendement financier. Les cinq caractéristiques ont pour année de base l'an 1989, l'année précédant immédiatement la période de l'étude.

Les entreprises étudiées ont été réparties selon leur actif total et leurs ventes respectivement. Ensuite, elles ont été regroupées par catégories de 10 millions de dollars pour chacune de ces deux caractéristiques de taille. Ceci révéla une grande dispersion dans la taille de ces entreprises par rapport à l'actif total et aux ventes. Plus d'un tiers des entreprises étudiées avaient un actif de moins de 20 millions de dollars, et plus d'un quart avaient des ventes inférieures à 20 millions de dollars. La dispersion était considérable et se ressemblait dans les deux catégories.

Puisque les résultats présentés dans les sections précédentes de cet article montrent qu'un pourcentage très élevé de répondants signalent des degrés importants de changement suivant la décision de leur compagnie de devenir publique, il est difficile de maintenir l'importance de la dimension "taille ». Évidemment, à moins qu'il ne soit argumenté que toutes les entreprises avec des ventes dépassant 10 millions de dollars peuvent être considérées comme étant de grande taille, et donc posséder "de facto " une structure formalisée, ces résultats indiquent que la dimension "devenir publique » peut être très importante pour expliquer les changements perçus par les trois groupes de répondants.

\section{Les effets du rendement financier}

La répartition statistique des caractéristiques reliées au rendement financier a aussi laissé voir une grande dispersion des entreprises étudiées. Le revenu de l'actif révélait que la majorité des entreprises avaient un rendement normal, avec plus de $60 \%$ rapportant des valeurs entre 0 et $10 \%$. Aucune différence majeure n'a été trouvée entre les deux groupes (A et B). Le revenu des actions a affiché une distribution plus uniforme avec $25 \%$ des entreprises étudiées affichant un retour au-dessus de $5 \%$. Les données sur les marges d'exploitation ont révélé un schéma semblable à celui dégagé par celles sur le coefficient de 
revenu de l'actif, c'est-à-dire qu'un pourcentage important des entreprises étudiées pouvaient être considérées comme ayant un rendement normal. Évidemment, il ne peut pas être maintenu que les résultats présentés dans les premières sections du présent travail reflètent seulement les perceptions de répondants venant d'entreprises qui réussissent. Les données sur les caractéristiques financières révèlent que les résultats de la recherche de Desroches et Jog (1989, 1991) ont plutôt été entrânés par la décision de l'entreprise de devenir publique. Il doit aussi être noté que les PDG et gestionnaires répondants montraient un degré significatif de mécontentement vis-à-vis l'évaluation boursière de leur entreprise, ce qui démontre que les résultats des questionnaires ont moins rapport au rendement financier, à la taille ou à l'évaluation de l'entreprise.

TABLEAU 9

Distribution des firmes de l'échantillon selon le retour sur l'actif (RSA)

\begin{tabular}{lrrrr}
\hline RSA & \multicolumn{2}{c}{ Groupe A* $^{*}$} & \multicolumn{2}{c}{ Groupe B** } \\
& $\mathbf{n}^{\text {* }}$ & \multicolumn{1}{c}{$\%$} & n $^{\text {* }}$ & \multicolumn{1}{c}{$\%$} \\
\hline Moins de 0,0 & 2 & 5,9 & 9 & 16,7 \\
PE 0,0 et MQ 5,0** & 10 & 29,4 & 16 & 29,6 \\
PE 5,0 et MQ 10,0 & 15 & 44,1 & 18 & 33,3 \\
PE 10,0 et MQ 15,0 & 4 & 11,8 & 7 & 13,0 \\
PE 15,0 et MQ 20,0 & 2 & 5,9 & 3 & 5,6 \\
PE 20,0 et MQ 25,0 & 1 & 2,9 & 1 & 1,9 \\
\cline { 2 - 5 } & 34 & 100 & 54 & 100 \\
\hline
\end{tabular}

* Firmes dont les répondants provenaient des trois niveaux, PDG, gestionnaires et employés.

** Firmes dont les répondants provenaient d'au moins un des trois niveaux.

*** $\mathrm{PE}=$ Plus grand ou égal à ; MQ = Moins que. 
TABLEAU 10

Distribution des firmes de l'échantillon selon le retour sur l'investissement (RSI)

\begin{tabular}{lcccc}
\hline $\begin{array}{l}\text { RSA } \\
(\%)\end{array}$ & $\mathbf{n}^{\circ}$ & Groupe A* & \multicolumn{2}{c}{$\mathbf{G r o u p e ~ B *}^{* *}$} \\
\hline Moins de 0,0 & 2 & 5,9 & 8 & $\mathbf{n}^{\circ}$ \\
PE 0,0 et MQ 5,0*** & 7 & 20,6 & 10 & 14,8 \\
PE 5,0 et MQ 10,0 & 5 & 14,7 & 6 & 18,5 \\
PE 10,0 et MQ 15,0 & 5 & 14,7 & 6 & 11,1 \\
PE 15,0 et MQ 0,0 & 5 & 14,7 & 7 & 11,1 \\
PE 20,0 et MQ 25,0 & 6 & 17,6 & 7 & 13,0 \\
PE 25,0 & 4 & 11,8 & 7 & 13,0 \\
& 34 & 100 & 54 & 100 \\
\hline
\end{tabular}

- Firmes dont les répondants provenaient des trois niveaux, PDG, gestionnaires et employés.

** Firmes dont les répondants provenaient d'au moins un des trois niveaux.

*** $\mathrm{PE}=$ Plus grand ou égal à ; MQ = Moins que.

\section{Conclusion}

Les résultats de recherche rapportés dans le présent travail indiquent que les processus de prise de décision et les structures des PME changent significativement suivant un premier appel à l'épargne publique. Les conclusions que l'on peut tirer de cette recherche à partir des perceptions passablement partagées par les PDG, les gestionnaires et les employés interrogés, sont d'une signification et d'une clarté remarquables.

Les entreprises examinées par cette recherche ont progressé vers une nouvelle phase de développement suivant leur passage au financement public. Le mouvement de ce que Thain et Mintzberg entre autres, ont nommé une «Phase 1 » (structure simple), vers une «Phase II » (structure fonctionnelle) a été accéléré par la décision de devenir entreprise publique.

Pour certains de ces changements, il est impossible de déterminer si la cause est l'accessibilité par la PME à une plus grande quantité de fonds, ou le fait d'avoir de nouveaux actionnaires externes qui peuvent exercer une influence directe et considérable sur les questions générales concernant la gestion de l'entreprise.

Les PME étudiées lors de cette recherche ont développé un processus de prise de décision plus décentralisée, sont devenues plus analytiques vis-à-vis la 
prise de décision, se sont formalisées dans leur structure, et ont élaboré des postes fonctionnels plus spécialisés. Tous ces changements corroborent un mouvement vers une organisation plus complexe et plus développée.

Pour un grand nombre de PME devenues publiques, des postes spécialisés ont aussi été créés afin d'accommoder une augmentation de liaisons externes. En particulier, des postes ont été créés pour veiller à la gestion des relations publiques et de la promotion, ainsi que pour régler les aspects légaux et les questions d'assurance. De fait, une spécialisation accrue dans ces domaines a été le changement le plus important dans ces entreprises par rapport à la création de nouveaux postes suivant l'appel à l'épargne publique.

Bien qu'il soit difficile de séparer de façon conclusive les effets du financement public des effets de simplement pouvoir disposer de plus de fonds, il semble que l'augmentation du degré de formalisation des entreprises examinées semble être plutôt le résultat de leur nouveau statut d'entreprise publique. Il est facile de constater que le développement d'une planification stratégique plus formalisée profiterait à ces entreprises en les aidant à présenter leurs plans futurs à leurs nouveaux actionnaires. Un accroissement de l'utilisation d'organigrammes hiérarchiques et de processus plus formels dans les domaines tels les relations avec les employés ont été découverts. Il est possible que dans ces domaines également la formalisation découle du nouveau statut d'entreprise publique, avec les nouvelles suggestions et contributions externes que celui-ci entraîne.

Un indice des effets du financement public sur la structure des objectifs des PME se trouve dans la manière qu'a augmenté l'emploi de techniques analytiques. Les deux sections où le changement fut le plus significatif ont été l'utilisation accrue de systèmes de comptabilité des responsabilités et partant, un meilleur emploi des centres de profit. Le fait que le rendement de ces organisations soit maintenant plus étroitement surveillé par des actionnaires externes pourrait expliquer ce mouvement vers un contrôle plus serré de la gestion et du rendement. 


\section{Bibliographie}

ChandleR, A.D. (1962), Strategy and Structure, Cambridge, M.I.T. University Press.

ChURChIL, N. et Y. Lewis (1983), "The five stages of small business growth ", dans Harvard Business Review, vol. 61, n 3, p. 30-51.

Covin, J.G. et D.P. SLevin (1988), « New venture competitive strategy : an industry life, cycle analysis ", dans Frontiers of Entrepreneurship Research, Wellesley, MA Babson College Center of Entrepreneurial Studies, p. 446-460.

Covin, J.G. et D.P. SLevin (1989), "Strategic management of small firms in hostile and benign environments », dans Strategic Management Journal, vol. 10, p. 75-87.

Desroches, J. et V. Jog (1989), « Les effets du financement public sur les entrepreneurs et leurs entreprises : Le cas des PME du Québec », dans la Revue internationale P.M.E., vol. 2, n 1, p. 19-37.

Desroches, J. et V. Jog (1991), La PME et le financement public : Attrait - décision impact, Halifax : L'Institut de recherches politiques, Canada, $155 \mathrm{p}$.

Desroches, J., V. JoG, et W. TAYLOR (1991), The Impact of Initial Public of Firms on $S M B$ 's, A Report submitted to the «Fonds F.C.A.R. », Québec, 75 pages plus annexes.

GOODING, R.Z. et J. WAGNER (1985), « A meta analytic review of the relationship between size and performance : the productivity and efficiency of organizations and their sub-units », dans Administrative Science Quaterly, vol. 30, p. 462-481.

HoFeR, C. et N.R. SANDBERG (1987), «Improving new venture performance : some guidelines for success », dans American Journal of Small Business, vol. 12, p. 11-25.

KuHN, R.L. (1982), Mid-Sized Firms : Success Strategy and Methodology, New York, Praeger.

March, I.J. et H.A. Simon (1958), Organizations, New York, John Wiley and Sons.

MaRsh, R.M. et H. MANNARI (1981), "Technology and organisational size as determinants of structure ", dans Administrative Science Quaterly, vol. 26, p. 33.

MIILeR, D. et P. FrIESEN (1983), « The life cycle in successfull and unsuccessful firms », dans Organization Studies, vol. 4, p. 339-356.

MilleR, D. et J.-M. Toulouse (1986), « Strategy, structure, CEO personality and performance in small firms », dans American Journal of Small Business, vol. 10, p. 47-62.

MiNTZBERG, H. (1979), The Structuring of Organizations, Englewood Cliffs, N.J., Prentice Hall.

Pugh, D.S., J.J. Hickson, C.R. Hinings et C. TuRnER (1968), « Dimensions of organization structure », dans Administration Science Quaterly, vol. 13, p. 65-105.

SANDBerg, W.R., New Venture Performance: The Role of Strategy and Industry Structure, Lexington, MA, Lexington Books.

Scort, B.R. (1971), Stages of Corporate Development, Case ${ }^{\circ}$ 9-371-294, Boston, Inter-Collegiate Case Clearing House, Harvard Business School.

ThaIN, D. (1969), «Stages of corporate development », dans Business Quaterly, hiver.

Toulouse, J.-M. (1980), Les réussites québécoises - défis des hommes d'affaires, Montréal, Les Éditions Agence d'Arc inc. 